\title{
Susy Hierarchies and Affine Lie Algebras
}

\author{
Francesco Toppan \\ Shizuoka University, Department of Physics, Ohya 836, Shizuoka city, Japan
}

\begin{abstract}
We review some basic features of the Lie-algebraic classification of $W$ algebras and related integrable hierarchies in $1+1$ dimensions, pointing out the role of affine Lie algebras. We emphasize that the supersymmetric extensions of the above construction possibly lead, though some questions are still opened, to the classification of supersymmetric hierarchies based on "generic" supersymmetric affine Lie algebras. Here the word generic is used to make clear that well-known procedures, as those introduced by Inami and Kanno, are too restricted and do not lead to the full spectrum of supersymmetric integrable hierarchies one can construct. A particular attention is devoted to the large- $N$ supersymmetric extensions (here $N=4$ ). The attention paid by large- $N$ theories being due to the fact that they arise as dimensional reduction of $N=1$ models, and moreover that they realize an "unification" of known hierarchies.
\end{abstract}

\section{Introduction}

In the last few years a lot of attention has been devoted to inter-related topics which go under the name of $W$-algebras, integrable hierarchies (non-relativistic) in $1+1$ dimensions of KdV or NLS type, 2-dimensional reativistic equations like Liouville (more generally Toda field theories) or SG, 0-dimensional matrix models which describe discretized 2-dimensional gravity.

Some of the above topics have definitely a more mathematical flavour, like for instance the theory and classification of $W$-algebras; some others are definitely more physically grounded, KdV describes waves in shallow water, Liouville equation is an ubiquous one, but at least a lot of attention has been paid to it in connection with non-critical strings.

Despite the fact that the above models and theories seem all very different and can be constructed in apparently unrelated ways it turned out indeed that they are just manifestations of an underlying mathematical framework. Indeed $W$-algebras (i.e. non-linear Poisson-bracket algebras of 1-dimensional fields containing a Virasoro one, which satisfy the standard properties of antisymmetry, Jacobi identity and Leibniz rule in the classical case) turn out to be the Poisson bracket structures for both relativistic Toda field theories and non-relativistic integrable equations like KdV, Boussinesq and so on. Moreover the Ward identities of generalized matrix models generate the so-called W-constraints and their partition functions turn out to be related to the $\tau$-functions of associated classical integrable hierarchies[1].

It deserves being mentioned that $W$-algebras themselves can be produced and classified via a truly algebraic approach, by putting restrictions to affine Lie algebras; such restrictions can be realized either as hamiltonian reductions or 
coset constructions (by looking at some centralizer over some enveloping algebra [2]). While there is maybe no strict mathematical proof so far that all $W$-algebras can be obtained with the methods of [3], at least there is no need to believe that all $W$-algebras cannot be obtained that way.

The production of such a closed structure like a $W$-algebra is an interesting mathematical activity by itself, however there is much more than that. A very peculiar and absolutely non-trivial feature of $W$-algebras arises when they allow constructing towers of infinite hamiltonians in involution. In this way they turn out to be linked to a dynamical system of a special kind, an integrable one. The technical tool which allows to prove integrability consists in formulating the dynamical system (and its associated $W$-algebra) via a Lax operator which can be either of scalar (KP-like) or matrix type.

In the next section we will sketch the main features of the bosonic construction, postponing to the later section the introduction of supersymmetric integrable systems with the necessary modifications.

\section{Bosonic Hierarchies}

Let us first point out that $2 D$ relativistic Toda models and $1+1$ non-relativistic integrable equations arise from constraining affine Lie algebras $\hat{\mathcal{G}}$ (and their associated enveloping algebras). The basic difference in the relativistic case is due to the fact that two copies of the affine algebra are considered, associated to the chiral and antichiral currents $J(z), \bar{J}(\bar{z})$ respectively. The dynamical fields are group-valued $g(z, \bar{z})$ and possibly expressed through a Gauss decomposition. We have

$$
J(z)=g^{-1} \partial_{z} g
$$

and a similar equation for $\bar{J}(\bar{z})$.

The simplest case is provided when $\mathcal{G}=s l(2)$. The three currents associated to $\operatorname{sl}(2)$ are $J_{ \pm}(x)$ and $J_{0}(x)\left(J_{0}(x)\right.$ generates the $U \hat{(1)}$ subalgebra).

In this simple case only two inequivalent constraints can be imposed on the (enveloping) affine algebra, either

A) constraining $J_{+}(x)=1$ (hamiltonian constrain), or

B) selecting the $X(y)$ centralizer of the enveloping algebra, namely

$\left\{J_{0}(x), X(y)\right\}=0$ (coset).

Accordingly, we get in the relativistic (I) and in the non-relativistic (II) cases the following dynamical systems:

I A) The Liouville equation.

II A) The m-KdV (and KdV) equation.

I B) The $2 D$ Witten's black hole.

II B) The Non-Linear Schrödinger Equation.

From now on we will concentrate only on the non-relativistic case, that is the system of integrable equations in $1+1$ dimension which can be solved through 
inverse scattering method. As mentioned above the integrability property is expressed by the fact that one can express the equations of motion through a Lax operator. We have two kinds of such operators, the scalar type

$$
L=\partial+\sum_{i=1, \ldots, \infty} u_{i} \partial^{-i}
$$

associated to the KP hierarchy, and the matrix type

$$
\mathcal{L}=\partial+\sum_{i} J_{i}(x) \tau^{i}+\Lambda
$$

where the currents $J_{i}(x)$ are valued in some Lie algebra $\mathcal{G}$ generated by $\tau^{i}$. $\Lambda$ is a constant element, depending on a spectral parameter $\lambda$, such that the loop algebra $\tilde{G}=\mathcal{G} \otimes C\left(\lambda, \lambda^{-1}\right)$ can be decomposed in the direct sum $\tilde{G}=K \oplus M$, with $K, M$ respectively the Kernel and Image under the adjoint action of $\Lambda$ (this technical property implies that $\mathcal{L}$ can be diagonalized under a similarity transformation).

In order to extract from scalar Lax operators integrable equations involving only a finite number of fields, we have to constrain the infinite fields $u_{i}(x)$ in a way consistent with the KP flows (constrained KP hierarchies). One possibility is requiring e.g. for a given $n L^{n}=L^{n}+$ (that is to be a purely differential operator). This is indeed a consistent constraint (leading to the $\mathrm{n}$-th $\mathrm{KdV}$ hierarchies), however it is known there exists many more inequivalent consistent constraints and a classification of them out of the scalar Lax operators alone appears rather impractical.

On the contrary it is well-known how to classify all possible hierarchies associated with affine algebras. They turn out to be related to the acceptable integral grading for any given loop algebra $\mathcal{G}$ and the choice of the regular element $\Lambda$ (see e.g. [4] for details). Moreover it is possible to relate such solutions with the constrained KP hierarchies. [5]

In order to be explicit we recall that in the original Drinfeld-Sokolov paper the n-th KdV hierarchies were obtained by assuming the underlying algebra to be $\operatorname{sl}(n)$ and the regular element $\Lambda$ to be the sum over the $s l(n)$ simple roots.

The above scheme seems quite satisfactory from the point of view of bosonic hierarchies since it provides a well-defined construction for them and is quite plausible they can all be accomodated in it. Questions concerning the possible equivalence of hierarchies arising from different choices of algebras, integral grading and/or regular element seem more tehnical and less central.

So far for purely bosonic hierarchies, in the next section we will introduce the supersymmetric ones.

\section{Supersymmetric Hierarchies}

The first natural question when discussing supersymmetric integrable hierarchies is of course why should we worry about them. One can think e.g. to the fact 
that so far no supersymmetric matrix model providing a discretized $2 D$ supergravity has been produced. Neverthless some achievement has been made like the introduction of a supereigenvalue model which is in a sense pull out of a hat but is related to a superintegrable hierarchy[6].

Morever the remarkable relation of KdV-type hierarchies with the conformal algebras (Virasoro and supersymmetric extensions) establishes a connection between such hierarchies and the (super-)string theories which has still to be fully appreciated.

From a purely mathematical point of view the role of supersymmetric integrable hierarchies and superalgebras is essential in at least two respects. Indeed, even when considering purely bosonic hierarchies, if we not allow for superstructures like super-algebras we cannot pretend to exhaust the full class of possible hierarchies, new integrable interacting purely bosonic hierarchies arise in fact from the bosonic sector (B-B and F-F submatrices) of supermatrix-valued superhierarchies.

Moreover investigating large $N$-extended superhierarchies corresponds to a sort of "unification or grandunification" program of known hierarchies. It happens in fact that unrelated bosonic hierarchies or lower supersymmetric $(N=$ 1,2 ) hierarchies turn out to be different manifestations of a single "unifying" large $N$ supersymmetric hierarchy. We will see later an example of this fact when discussing the $N=4 \mathrm{KdV}$ hierarchy.

The point of view that we adopt here in discussing supersymmetric hierarchies is based on the (super-)Lie algebra framework, which one can reasonably hope will provide the key to classify all superhierarchies. The main reason of the difficulty involved in classifying superhierarchies w.r.t. the purely bosonic ones is due to the complications involving the presence of both even and odd generators.

We need to point out that (super)-Lie algebras appear in 3 different classes according whether they admit a presentation in terms of Dynkin diagrams with simple roots which are either:

i) purely fermionic,

ii) necessarily of mixed type, or

iii) purely bosonic (they are reduced to standard Lie algebras).

A simple argument made people believe for a long time that only the special class of super-Lie algebras admitting fermionic simple roots were relevant for the construction of superhierarchies. Inami and Kanno [7] gave it in the contest of super-KdV hierarchies. In order to extend the bosonic matrix Lax operator they were led to consider a supersymmetric Lax of the kind

$$
\mathcal{L}=D+\Psi(X)+\Lambda
$$

where now $D$ is the $(N=1)$ fermionic supersymmetric derivative. $\Psi(X)$ are superfields valued in some superalgebra and as such are fermionic. The regular element $\Lambda$ should be given by the sum over the simple roots and in order to respect statistics it must be fermionic as well. Therefore it seemed that only class i) superalgebras had to be considered. A similar argument was given by Evans 
and Hollowood [8] in the case of superToda theories. This argument has paved the way to the standard supersymmetrization recipe of bosonic models which goes as follows: embed the given bosonic algebra which provides the bosonic system into a larger superLie algebra having "good properties" and perform the hamiltonian reduction on it. In this way $N=1$ extension of $\mathrm{KdV}$ and Liouville equations were provided in terms of the $\operatorname{osp}(1 \mid 2)$ superalgebra.

The Inami-Kanno scheme is a perfectly consistent one, leading to a classification of supersymmetric hierarchies much similar to the bosonic case. There would be no need to look for improving it if it would not turn out a too restricted one. Indeed it happens that well-known and interesting superhierarchies cannot be accomodated in it. To my knowledge Brunelli and Das were the first [9] who faced this problem when they realized that the superNLS equation admits a Lax operator based on the $\operatorname{sl}(2)$ bosonic algebra (and not $\operatorname{ssp}(1 \mid 2)$ as one would have been expected). In [10] it was pointed out that the superNLS hierarchy arises as a coset construction (just as its bosonic counterpart) over a Poisson bracket structure based on the superaffinization (that is expressed in terms of superfields) of the bosonic $\operatorname{sl}(2)$ algebra.

As a consequence there exists a much bigger class of supersymmetric integrable models than previously expected which need to be investigated. Despite the fact that we do not have yet a systematic way of constructing them in terms of matrix Lax operators just like the bosonic models or the Inami-Kanno superhierarchies, still we can develop some strategy to investigate them. This will be explained next.

\section{Supersymmetric Hierarchies and Affine Algebras}

Let us here discuss a possible strategy for constructing supersymmetric integrable hierarchies from generic superaffinizations of (super-)Lie algebras. But first let us point out that a superaffinization of a given (super-)Lie algebra $\mathcal{G}$ with generators $\tau^{i}$ and structure constants $f^{i j}{ }_{k}$ is realized by $N=1$ superfields $\Psi^{i}(X)$, with opposite statistics w.r.t. $\tau^{i}$ and such that

$$
\left\{\Psi^{i}(X), \Psi^{j}(Y)\right\}=f^{i j}{ }_{k} \Psi^{k}(Y) \delta(X, Y)+K^{i j} D_{Y} \delta(X, Y)
$$

with $\delta(X, Y)$ the $N=1$ delta-function and $K^{i j}=\operatorname{Str}\left(\tau^{i} \tau^{j}\right)$ in some given (let's say the adjoint) representation of $\mathcal{G}$.

The following steps should be performed:

i) Take a superaffine (super-)Lie algebra which should be regarded as Poisson bracket structure.

ii) Make some Ansatz over the possible hamiltonians in involution; this would mean imposing symmetry requirements, cosets or hamiltonian reductions.

iii) Check the consistency of flows and if indeed at lower orders one gets hamiltonians in involution.

iv) Try to figure out the form of possible Lax operators (this is the most difficult task). 
It can even happen that one finds more structures than expected. Indeed it is well-known that a relation exists between division algebras ad extended supersymmetries. Complex, Quaternionic ad Octonionic structures are associated to (global) $N=2,4,8$ extensions respectively.

A complex structure for a (super-)algebra over the real fields is an operation $J$ which satisfy $J^{2}=-1$, while a quaternionic structure involves 3 complex structures $J_{i}$ whose mutual algebra is that of the Pauli matrices.

If a theory admits a complex structure it necessarily has an extended supersymmetry. For instance the superNLS equation which arises from the $s \hat{l}(2) / u \hat{(1)}$ structure is automatically $N=2$ since such a coset admits a complex structure (while $s l(2)$ does not). An elegant (but equivalent) formulation can be realized through the coset $\operatorname{sl}(2) \hat{\oplus} u(1) / u(1) \hat{\oplus} u(1)[11]$. This construction allows a manifestly $N=2$ superfield formulation since the extra $u(1)$ are added to give a complex structures for both numerator and denominator.

The $\operatorname{sl}(2) \oplus u(1)$ algebra turns out to be vey interesting because it appears in the list given by [12] (actually these authors considered group-manifolds, out of which algebras can be immediately recovered) as the simplest example of non-abelian algebra (the even simpler abelian case being $u(1)^{\otimes 4}$ ) admitting a quaternionic structure.

A natural question therefore arises, namely if it is possible that the superaffine algebra $s l(2) \hat{\oplus} u(1)$, taken as a Poisson bracket algebra, would allow to play another game, not just the coset already mentioned, according to the above scheme. In particular we can ask ourselves if we can demand an $N=4$ symmetry requirement which in turns imply an $N=4$ hierarchy. In the next section we will show that this is indeed the case [13].

\section{5 the $N=4$ structure of $\operatorname{sl}(2) \oplus u(1)$}

The superaffine algebra $s l(2) \hat{\oplus} u(1)$ can be conveniently described in terms of $N=2$ superfields. Let us introduce the $N=2$ fermionic derivatives $D, \bar{D}$ whose algebra reads as follows

$$
\begin{aligned}
D^{2}=\bar{D}^{2} & =0 \\
\{D, \bar{D}\} & =-\partial_{x}
\end{aligned}
$$

The spin $\frac{1}{2} N=2$ superfields are denoted as $H, \bar{H}, F, \bar{F}$.

$H$ and $\bar{H}$ are associated to the $u(1) \oplus u(1)$ subalgebra.

They are constrained superfields, the constraints being non-linearly realized

$$
\begin{aligned}
& D H=\overline{D H}=0 \\
& (D+H) F=(\bar{D}-\bar{H}) \bar{F}=0
\end{aligned}
$$

The non-vanishing structure constants are given by

$$
\{H(1), \bar{H}(2)\}=D \bar{D} \delta
$$




$$
\begin{aligned}
& \{H(1), F(2)\}=D F \cdot \delta \\
& \{H(1), \bar{F}(2)\}=-D \bar{F} \delta \\
& \{\bar{H}(1), F(2)\}=-\bar{D} F \cdot \delta \\
& \{\bar{H}(1), \bar{F}(2)\}=\overline{D F} \cdot \delta \\
& \{F(1), \bar{F}(2)\}=(D+H)(\bar{D}+\bar{H}) \delta+F \bar{F} \delta
\end{aligned}
$$

where $\delta \equiv \delta(1,2)$ is the $N=2$ delta-function and the derivatives in the r.h.s. are computed at $Z \equiv 1$.

In the last line a "fake" non-linear term appears. It is not present when the chiral constraints are solved in terms of $N=1$ superfields or component fields.

There exists a second set of global $N=2$ non-linear supersymmetries, expressed through the infinitesimal parameters $\epsilon, \bar{\epsilon}$, which results from the quaternionic structure associated with $s l(2) \oplus u(1)$. We have

$$
\begin{aligned}
\delta H & =\epsilon D \bar{F}+\bar{\epsilon} H F \\
\delta \bar{H} & =\bar{\epsilon} \bar{D} F-\epsilon \overline{H F} \\
\delta F & =-\epsilon D \bar{H}-\epsilon(H \bar{H}+F \bar{F}) \\
\delta \bar{F} & =-\bar{\epsilon} \bar{D} H-\bar{\epsilon}(H \bar{H}+F \bar{F})
\end{aligned}
$$

It can be easily checked that the above transformations preserve the chirality constraints and that their commutators close to give, together with the original transformations, an $N=4$ supersymmetry.

\section{6 the $N=4$ Hierarchy}

We have seen that $s l(2) \hat{\oplus} u(1)$ carries an $N=4$ structure. To prove the existence of globally invariant $N=4$ dynamical systems we have to construct explicitly the $N=4$ invariant hamiltonians. They indeed exist and moreover, at the lower dimensional integral spin dimension $d=1,2$, they are unique up to total derivatives (at least if a global chargeless condition is required, where $H, \bar{H}$ are chargeless, while $F$ and $\bar{F}$ have charges +1 and -1 respectively).

We have indeed

$$
\begin{aligned}
H_{1}= & F \bar{F}+H \bar{H} \\
H_{2}= & F^{\prime} \bar{F}-H^{\prime} \bar{H}- \\
& -(D \bar{H}+\bar{D} H)(H \bar{H}+F \bar{F})-2 H \bar{H} F \bar{F}
\end{aligned}
$$

Higher dimensional $N=4$ hamiltonians can be explicitly constructed and turn out to be in involution with the lower dimensional ones.

The resulting equations of motion (which is not needed to write here,see [13]) with respect to the second hamiltonian realize an $N=4$ dynamical system which combines in a non-trivial way both the $N=2 \mathrm{mKdV}$ equation and the $N=2$ NLS equations. The latters are recovered by setting, consistently with 
the equations of motion, respectively $F=\bar{F}=0$ and $H=\bar{H}=0$. A third, more mysterious, $N=2$ system can be obtained by performing a non-symmetrical reduction leading to $\bar{H}=F=0$.

So far for what concerns the construction of the $N=4$ system. An Ansatz has guided us towards its realization and we have seen that it is essentially unique. A point which has been left apart consists in explicitly proving that our system indeed corresponds to an integrable hierarchy admitting an infinite tower of hamiltonians in involution. In this particular case we have a very elegant procedure which proves that. Unfortunately, as already stated, we cannot rely so far on any systematic construction for the Lax operators valid for generic theories. The best we can do at present is based on a trial procedure.

However, for the $\operatorname{sl}(2) \hat{\oplus} u(1)$ case the key property which allows to solve the problem is the existence of a (differential polynomial) $N=4$ Sugawara construction [13]. This very remarkable transformation has at least four different consequences:

i) it provides a linearization of the $N=4$ transformations,

ii) it furnishes a realization for the "minimal" $N=4$ SuperConformal Algebra (SCA),

iii) it relates the "affine hierarchy" to the $N=4 \mathrm{KdV}$ system[14] and

iv) it allows the construction of the Lax operator which proves the integrability.

The Sugawara transformation is a differential polynomial transformation which express the "superconformal fields" through the original affine superfields $H, \bar{H}, F, \bar{F}$. The transformed superfields are an $N=2$ real superVirasoro superfield $J$ (with component fields content $\left(1, \frac{3}{2}, \frac{3}{2}, 2\right)$ ) plus two chiral and antichiral spin 1 superfields (in components $\left(1, \frac{3}{2}\right)$ ). We have explicitly

$$
\begin{aligned}
& J=H \bar{H}+F \bar{F}+D \bar{H}+\bar{D} H \\
& \Phi=D \bar{F} \\
& \bar{\Phi}=\bar{D} F
\end{aligned}
$$

The presence of the Feigin-Fuchs terms in the r.h.s. for $J$ is especially important. Without them $J$ would be a nilpotent field $\left(J^{3}=0\right.$ due to the fermionic character of $H, \bar{H}, F, \bar{F}$ ). Moreover they allow the second set of $N=2$ transformations to close linearly on $J, \Phi, \bar{\Phi}$ as

$$
\begin{aligned}
& \delta J=-\bar{\epsilon} D \bar{\Phi}-\epsilon \bar{D} \Phi \\
& \delta \Phi=\bar{\epsilon} D J \\
& \delta \bar{\Phi}=\epsilon \bar{D} \Phi
\end{aligned}
$$

The composite superfields $J, \Phi, \bar{\Phi}$ satisfy a closed algebra structure under the original $s l(2) \hat{\oplus} u(1)$ Poisson bracket structure and it coincides with the minimal version of the $N=4$ SCA. 
The hamiltonians in involution can be closely expressed through the superfields $J, \Phi, \bar{\Phi}$ alone. At the lowest order we have, for the hamiltonian densities

$$
\begin{aligned}
& H_{1}=J \\
& H_{2}=J^{2}-2 \Phi \bar{\Phi} \\
& H_{3}=J[D, \bar{D}] J+2 \Phi \bar{\Phi}^{\prime}+\frac{2}{3} J^{3}-4 J \Phi \bar{\Phi}
\end{aligned}
$$

As a consequence we have a closed system of dynamical equations for $J, \Phi, \bar{\Phi}$ which coincides with the $N=4 \mathrm{KdV}$ hierarchy.

The Lax operator can be borrowed from the known Lax operator of KdV and is given by [15]

$$
L=D \bar{D}+D \bar{D} \partial^{-1}\left(J+\bar{\Phi} \partial^{-1} \Phi\right) \partial^{-1} D \bar{D}
$$

It should be noticed that in this particular case checking the integrability properties of the given hierarchy was immediate once the Sugawara construction has been taken into account since the above Lax operator for the $N=4 \mathrm{KdV}$ was already known. However, even if this would have not been the case (as one could expect from constructions based on more general algebras), the Sugawara transformation itself would greatly simplify the task of finding the correct Lax pair, since it is much easier to deal with three spin 1 fields than with spin $\frac{1}{2}$ superfields. The dramatic simpification of the hamiltonians when expressed through $J, \Phi, \bar{\Phi}$ is also an example.

Some more comments are in order: the $N=4 \mathrm{KdV}$ is the "unifying hierarchy" for two of the three inequivalent $N=2 \mathrm{KdV}$ hierarchies labeled by $a=1,-2,4$. The $a=-2$ and $a=4 \mathrm{~N}=2 \mathrm{KdV}$ are indeed obtained from different reductions of $N=4 \mathrm{KdV}$.

The construction based on the abelian $u(1)^{\otimes 4}$ algebra could lead to global $N=4$ hierarchies realized through strictly chiral and antichiral superfields, but it can be easily checked that they are definitely not polynomial generalization of $N=2$ NLS and are not $N=4$ superconformal.

\section{Conclusions}

We have pointed out that supersymmetrical integrable hierarchies can be very naturally investigated (and hopefully classified) taking as a starting point the (super-)Lie algebras and their supersymmetric affinizations. Our approach is very much complementary with the point of view advocated by many authors in literature (like e.g. Z. Popowicz who is also author of a package for computing Lax operators by using reduce). They rather use the converse attitude of actually producing integrable equations in terms of some consistent Lax operators, especially of scalar type. This approach has the advantage of furnishing indeed integrable systems, but leave aside questions concerning the algebraic interpretation of these results. The approach based on Lie algebras has just opposite merits and drawbacks. It furnishes from the very beginning the algebraic 
setting for defining dynamical systems and provides guidelines how to obtain them, while the burden is on proving the existence of a tower of hamiltonians in involution.

This situation is very specific to the supersymmetric case since, in contrast with bosonic hierarchies, we do not dispose of a hamiltonian reduction procedure which automatically leads to Lax operators. The examples where this is indeed the case, corresponding to the Inami-Kanno hierarchies, are of interest but they belong to a restricted class. Other interesting super-integrable systems like the $N=4 \mathrm{KdV}$ equation previously discussed are left out of this scheme.

The approach based on (super-)Lie algebras is a very convenient one in the investigation of supersymmetric extended hierarchies. As discussed in this paper, one has to look for algebras admitting extra structures, complex, quaternionic and so far. Some partial results obtained in collaboratin with Ivanov and Krivonos show indeed that $\operatorname{sl}(3)$, the next simplest quaternionic algebra, admits a global $N=4$ structure which suggests the realization of at least global $N=4$ hierarchies.

In conclusion it deserves being mentioned that investigating supersymmetric integrable hierarchies looks promising due to the presence of open problems.

\section{Acknowledgments}

This work has been done under JSPS contract. The author expresses its gratitude to the members of the Phys. Dept. of Shizuoka University for their kind hospitality.

\section{References}

1 P. Di Francesco, P. Ginsparg, J. Zinn Justin, Phys. Rept. 254, 1 (1995)

2 F. Delduc, L. Frappat, P. Sorba, F. Toppan, E. Ragoucy, Phys. Lett. B 318, 457 (1993)

3 L. Frappat, E. Ragoucy, P. Sorba, Comm. Math. Phys.157, 93 (1993)

4 I. R. McIntosh, . An Algebraic Study of Zero Curvature Equations Ph.D. Thesis, Dept. Math., Imperial College, London (1988), unpublished; M.F. de Groot, T.J. Hollowood, J.L. Miramontes, Comm. Math. Phys. 145, 57 (1992); L. Feher, J. Harnad, I. Marshall, Comm. Math. Phs. 154, 181 (1993)

5 H. Aratyn, J.F. Gomes, A.H. Zimerman, J. Math. Phys. 36, 3419 (1995): H. Aratyn, E. Nissimov, S. Pacheva, Int. Jou. Mod. Phys. A 12, 1265 (1997)

6 L. Alvarez-Gaume', H. Itoyama, J.L. Manes, A. Zadra, Int. Jou. Mod. Phys. A 7, 5337 (1992)

7 T. Inami, H. Kanno, Comm. Math. Phys. 136, 519 (1991); Int. Jou. Mod. Phys. A 7 Suppl. 1A, 419 (1992); Jou. Phys. A 25, 3729 (1992)

8 J. Evans, T. Hollowood, Nucl. Phys. B 352, 723 (1991)

9 J.C. Brunelli, A. Das, Phys. Lett. B337, 303 (1995); Jou. Math. Phys. 36, 268 (1995)

10 F. Toppan, Int. Jou. Mod. Phys. A 10, 895 (1995)

11 S. Krivonos, A. Sorin, F. Toppan, Phys. Lett. A 206, 146 (1995) 
12 P.H. Spindel, A. Sevrin, W. Troost, A. Van Proeyen, Phys. Lett. B 206, 71 (1988)

13 E. Ivanov, S. Krivonos, F. Toppan, Preprint hep-th/9703224 (1997), to appear in Phys. Lett. B

14 F. Delduc, E. Ivanov, Phys. Lett. B 309, 312 (1993)

15 F. Delduc, L. Gallot, E. Ivanov, Preprint hep-th/9611033 (1996) 